\title{
Wireless Technologies, Ubiquitous Computing and Mobile Health: Application to Drug Abuse Treatment and Compliance with HIV Therapies
}

\author{
Edward W. Boyer • David Smelson • Richard Fletcher • \\ Douglas Ziedonis • Rosalind W. Picard
}

Published online: 10 July 2010

(C) American College of Medical Toxicology 2010

\begin{abstract}
Beneficial advances in the treatment of substance abuse and compliance with medical therapies, including HAART, are possible with new mobile technologies related to personal physiological sensing and computational methods. When incorporated into mobile platforms that allow for ubiquitous computing, these technologies have great potential for extending the reach of behavioral interventions from clinical settings where they are learned into natural environments.
\end{abstract}

Keywords Drug abuse $\cdot$ Medication compliance $\cdot$ Ubicomp

\section{Introduction}

Mobile health ("mHealth") is the use of mobile technologies as a platform for health research and healthcare delivery. mHealth applications include public health education, remote data collection, point-of-care diagnostics, remote patient monitoring, and promotion of healthy behaviors. In the paper, we describe mobile technologies

\section{E. W. Boyer $(\square)$}

Department of Emergency Medicine,

University of Massachusetts Medical School,

55 Lake Avenue North,

Worcester, MA 01655, USA

e-mail: edward.boyer@childrens.harvard.edu

D. Smelson $\cdot$ D. Ziedonis

Department of Psychiatry,

University of Massachusetts Medical School,

Worcester, MA, USA

R. Fletcher $\cdot$ R. W. Picard

Media Lab, Massachusetts Institute of Technology,

Cambridge, MA, USA that incorporate state-of-the-art wireless connectivity, personal sensing, and machine learning approaches that are intended to intervene in health risk behaviors in populations of considerable public health importance.

Part I: mHealth Approaches to Treatment of Substance Abuse

Comparison of Drug Addiction to Chronic Illness Addiction can be defined as the compulsive use of drugs despite negative consequences [1]. Some persons successfully stop problematic substance use, but for a large proportion of affected individuals, addiction is a relapsing, chronic condition [1]. Increasingly, clinicians recognize that drug addiction shares characteristics with other chronic medical illnesses. For example, the course of addiction, like that of Type II diabetes and hypertension, has the potential to respond to lifestyle modification [2]. Where pharmacological therapies for addictive diseases are available, approximately $40-60 \%$ of addicted patients adhere to therapy; relapse - a change in condition that requires a patient receive medical care to reestablish remission of symptoms - occurs in the same proportion. These numbers are similar to other chronic diseases such as diabetes, hypertension, and asthma, and raise questions regarding current treatment of addictive illness [2].

Contrast Between Treatment Approaches to Drug Addiction and Chronic Illness Although drug addiction has many similarities to chronic illness, the available options for treatment are dramatically different. Unfortunately, most treatments for addiction lack the ongoing monitoring applied to chronic diseases; the treatment approach for addiction, therefore, often resembles that for acute illnesses 
in which patients receive detoxification only until the acute withdrawal symptoms subside. Other patients who are admitted to specialty drug treatment programs undergo rehabilitation for excessive drug use and are then discharged into environments themselves associated with drug use [2]. The lack of treatment continuity is a significant problem in substance abuse treatment even though evidence indicates that patients who remained engaged in care such as behavioral therapy, 12-step support groups or participating in methadone maintenance therapy have shown success in preventing relapse $[3,4]$.

In addition, entry into drug treatment programs is very different for problematic substance use than for other chronic diseases. Patients who develop conditions such as congestive heart failure, chronic obstructive pulmonary disease, and type II diabetes mellitus typically exhibit predicable progressions of illness that are diligently monitored by clinicians; once patients reach the diagnostic criteria for a particular disease, they are often referred to a specialist for more focused management. Individuals enter drug treatment programs differently. Often in the context of acute intoxication, in the accompaniment of law enforcement, or between the hours of 430 P.M.-9 A.M., individuals with drug addiction present for care through emergency departments. While emergency physicians are adept at managing acute drug overdose and intoxication, emergency departments are often crowded and lack the resources to provide staff to comprehensively evaluate problematic substance use, arrange entry to drug treatment, or establish case management follow-up. Consequently, patients are often discharged from emergency departments with little if any attention paid to developing a treatment plan. Addicted individuals who seek drug treatment at night or during weekends may paradoxically relapse because they cannot endure waiting for business hours for treatment centersand the treatment they offer- to open.

Understanding Drug Abuse and Relapse Several theories have been proposed to explain this persistent desire and relapse to substance use after periods of abstinence. These include behavioral theories of choice, which view relapse as the outcome of a decision-making process weighing immediate versus long-term rewards, and cognitive social learning theory, in which relapse results from a perceived lack of personal control and specific cognitive-affective reactions to initial slips [5]. A third approach is derived from classical conditioning theory, which holds that relapse occurs when situational events or cues elicit the motivational state underlying the substance use behavior [5, 6]. While earlier conditioning theorists believed that counter conditioning and extinction would destroy previously learned addictive behaviors, current researchers adhere to a more complex model, which holds that addictive behavior can be reinstated following extinction by exposure to drugs, drug-related cues, or environmental stressors [6-8]. Knowing individual affective responses (e.g., the desire to use drugs) to events in natural environments (e.g., drug use cues) as they occur is therefore important to an improved understanding of relapse, the development of prevention strategies, and interventions for risk reduction.

The Need for Better Tools for Assessment and Intervention Unfortunately, the absence of a "gold standard" to accurately assess changes in risk behavior remains a key obstacle in evaluating outcomes of health risk prevention studies. One approach involves the collection of selfreported behavioral data, often by retrospective assessment using face-to-face interviews, self-administered questionnaires, and computer-assisted self-interviews. Perhaps the most valid method available for evaluating the accuracy of retrospective self-reported information is a within-subjects comparison of these data to information recorded prospectively in diaries over the same time period.

Despite its perceived validity, self-reported information suffers from inaccurate or untruthful reports of recalled behavior, self-presentation response distortions, comprehension, or memory failure. These problems can occur in any self-recall setting but are particularly acute in addictive disorders in which denial, minimization, and rationalizations are common. To circumvent these shortcomings, experience sampling methods such as ecological momentary assessment (EMA) have been developed [9]. Using fixed or random prompts that interrupt treatment participants several times a day, EMA methods are designed to obtain "real time" behavioral data into a diary to capture processes like coping, emotion regulation, behaviors, and contexts - things that recall-based questionnaires do not evaluate well. Traditional EMA protocols often miss precise environmental and behavioral events and are so highly disruptive that EMA observation periods are limited to only a few days. An improved understanding of environmental and behavioral triggers for relapse is needed, particularly for abuse to adrenergic drugs such as cocaine and methamphetamine where up to $70 \%$ of treated patients returned to heavy use within the year [10].

The Need for Better Tools to Support Behavioral Interventions Currently available tools for drug abuse interventions remains limited. For example, psychosocial treatments for cocaine addiction remain the cornerstone of therapy since no FDA approved medication options exist. Unfortunately, patients who receive behavioral interventions in sterile clinical environments such as clinics are often unable to enact these therapies in the real world. Consequently, developing methods to extend the reach of behavioral therapies beyond the narrow confines of an office setting 
would potentially improve treatment compliance and patient health at greatly reduced cost.

To address the need for better intervention tools, the National Institutes of Health has declared investigations that deliver evidence-based behavioral modification interventions using mobile technologies are a research priority. Although the use of mobile computing devices such as PDAs and cellphones to affect behavior has increased dramatically, their value to date remain unproven [11]. For example, at least 15 "read-only" smoking cessation applications are commercially available for use on Apple's iPhone, as are 12 weight loss programs. None of these applications, however, describes the behavioral theory underlying the program, and none has withstood any testing for effectiveness. The major failing of read-only devices is that behavior arises from the milieu of neurobiological, physiological, cultural, and environmental processes; without measurement of emotions, physiology, and personality, portable computing devices are unable to interpret behavior and intervene in problematic substance use, addiction, and relapse [12]. In addition, the use of messaging prompts requires that the person carrying the device be interrupted to provide information on stress, anger, frustration, and feeling deprived affect substance abuse and relapse [13]. Furthermore, the annoyance of devices that interrupt may limit their acceptance and contribute to a sense of loss of control that may itself exacerbate the need to use drugs.

\section{A New Prescription for Behavior Modification Effective} tools for understanding and affecting human behavior thus require simultaneous measurement of co-occurring behavioral and biological variables and must occur in a "real time" continuous, unobtrusive, and objective manner. Furthermore, they must have the capability to be individualized, to adapt to rapid changes in patient condition, and deliver interventions on an ongoing basis. The technologies for realizing such systems are now emerging.

Miniature wearable sensors that are now available measure biological changes in heart rate and skin conductance which are associated with autonomic nervous system and affective response [14]. If well conceived and implemented, mobile computing devices that detect behavioral states can lead to highly individualized interventions for substance abuse and relapse prevention. Furthermore, while most individuals do not confide in others - including familywhen cravings develop and may not even be aware of the signs and symptoms that precede relapse, such technologies can be used to deliver interventions precisely at the moment of greatest need.

The timing of an intervention is, however, a complex task. A sensor-laden wristband, for example, can automatically measure physiological signs, but detection of drug craving requires modeling of human behavior, a complex dynamic system. Fortunately, machine learning-a branch of artificial intelligence-represents an ideal solution to this problem. Machine learning algorithms learn from a limited number of examples where the data may be noisy and contain complex patterns which elude human detection. One approach, Dynamic Bayesian Networks (DBNs), can be used to discover patterns of physiologic, affective, and contextual measures that predict health risk behavior [15]. DBNs are an application of Bayesian Networks to timevarying problems, where the relationships between variables are replicated at each time step [16]. As the name suggests, DBNs are a natural choice to model a complex dynamic system such as behavioral states confounded by time-varying comorbidities that may come into play in the moments before relapse. Since DBNs are designed to manage noisy data, unknown quantities and uncertain events, they have the power to describe not only instantaneous correlations among variables, but also how their values change over time. DBNs can generalize from limited data because the learning algorithm stresses balancing performance with model complexity. An overly complex model might be able to explain a data set (such as continuous physiology monitoring data) perfectly, but fails to generalize because it is explaining the data's idiosyncrasies (e.g., the humidity that day) of the specific data set. By penalizing model complexity, the algorithm finds the simplest acceptable explanation of the patterns - which are more robust to noise in existing data and tend to generalize better to future data. Once the DBN has learned to identify a drug use trigger or episode, it can instruct the device to trigger a multimedia message designed to counter cravings. The same system can also be used to automatically send a text message to the person's caregiver or support group to request assistance.

The use of mobile computing devices and wearable sensors, therefore, offers what motivational enhancement therapy, relapse prevention, and 12-step facilitation cannot do alone - provide ongoing assessment, personalized feedback, and "in vivo" clinical support for $24 \mathrm{~h} /$ day, 7 days/ week. The ability to provide timely personalized feedback based on biological and behavioral processes, currently unachievable in traditional psychosocial drug abuse treatment interventions, would thus be an important innovation and advancement for treatment providers.

\section{II. mHealth Approaches to HIV Compliance}

The Importance of Compliance with HIV Medications Since its introduction in 1996, highly active antiretroviral therapy (HAART) has become standard of care for HIV treatment by reducing viral load and decreasing HIV-related mortality 
[17]. Unfortunately, the unique characteristics of the HIV virus require near-perfect adherence to multidrug antiretroviral regimens to achieve the maximal suppression of viral replication and prevent drug resistance. Patients who do not strictly adhere to treatment regimens can expect viral drug resistance as well cross-resistance that makes therapies ineffective. Even worse, a drug-resistant strain of HIV caused by noncompliance can be transmitted to an uninfected person who then has few, if any, effective treatment options [17].

A direct association exists between poor compliance and the complexity of antiretroviral regimens, the number of prescribed medications, and the extent to which medication regimens interfere with daily lives; in fact, difficulties with simply taking the medications, not their effects, are the most consistent findings from research on compliance [18]. Multidrug HAART regimens have been among the most complicated open-ended therapies that have been devised since the timing of meals and amount of fluid intake, in addition to the medication schedule, are considered important for optimal outcomes. Even on its most basic level, the sheer number of prescribed medications can be breathtaking: HIV-positive patients can take up to 15 medications per day [19]. The medications taken by many HIV-positive patients to prevent opportunistic infections, stimulate appetite, treat pain and depression, and treat insomnia add additional layers of treatment complexity. Finally, many individuals take OTC medications to treat therapy side effects such as nausea and diarrhea, ingest herbal products and dietary supplements, or even take experimental therapies.

The extent to which patients are noncompliant is important in preventing outgrowth of resistant viral strains. For example, $81 \%$ of patients with $95 \%$ adherence demonstrated viral suppression, but only half of individuals who were $80-90 \%$ compliant were successful [17]. Only $6 \%$ of patients who were $70 \%$ compliant had improvements in viral load [17]. These sobering suppression rates highlight the urgent need for improved interventions to improve compliance with HAART therapies.

HAART Therapy may be Effective in Drug Using Populations Although many treatment experts recognize that HAART is most effective in populations that abstain from illicit drugs, the therapies may be effective in HIV+ populations with that continue to use drugs. For example, injection drug users (IDUs) receiving HAART therapy may realize the comparable survival outcomes as non-IDUs receiving the same medications [20]. In both populations, adherence to HIV treatment outcomes must be precise, consistent and rigorous, since less-than-optimal adherence leads to viral resistance and quicker disease progression. In
IDU populations, adherence is extremely poor, with estimates of between $25 \%$ and $50 \%$ of patients failing to follow treatment guidelines. The frequency of drug use appears to have minimal impact on HAART noncompliance; for example, HAART noncompliance in $\mathrm{HIV}+$ men who have sex with men was related to the use of methamphetamine, not the number of times or the amount of methamphetamine use [21].

\section{Technological Approaches to HAART Compliance}

Most literature on the use of technologies to monitor adherence to HAART has not included active drug users. In addition, most of the adherence data from HIV + drug users are retrospective; it is therefore impossible to determine methods to intervene as nonadherent behaviors arise. Nonetheless, advanced technologies have been successfully deployed in drug using populations. EMA and electronic diary protocols in methadone users successfully recorded cue exposure, craving, and mood in the hours before cocaine and/or heroin use [22]. Sufficient precedent therefore exists for the use of technological instrumentation, computer software, and other mHealth technologies that can simultaneously monitor and assess adherence in HIV+, drug-using populations.

\section{Theoretical Bases of Technology-based HAART Compliance Interventions}

Several theoretical models have been used as a basis for technology-delivered interventions; most of these are applicable to enhancing compliance with HIV medications. Blended therapies based upon Motivational Enhancement Theory, Motivational Interviewing, and Social Cognitive Theory perhaps integrate best with ubiquitous technologies [23]. These theories have been the bases for highly effective interventions such as Life-Steps [24]. Internet-delivered interventions have drawn from the Information-MotivationBehavior model, as well as the Stage of Change and Social Learning Theories [25-27]. Finally, the Health Belief Model and Theory of Reasoned Action have been applied to technology-based interventions directed toward numerous diseases such as hypertension, diabetes, coronary artery disease, and HAART compliance. Despite many years of focused research, however, one major problem of existing HAART compliance interventions is that they extinguish once the intervention is removed, losing effectiveness over time. An urgent need therefore exists for novel, multimedia interventions that preserve their effectiveness even after the completion of a study. 


\section{Conclusions}

Beneficial advances in the treatment of substance abuse and compliance with medical therapies, including HAART, is possible with new sensor and computational technologies. When incorporated into mobile platforms that allow for ubiquitous computing, these devices have great potential extending the reach of clinic-based interventions and therefore hold promise for realizing true behavior modification.

\section{References}

1. Hyman S (2005) Addiction: a disease of learning and memory. Am J Psychiatry 162:1414-1422

2. McLellan A, Lewis D, OBrien C, Kleber H (2000) Drug dependence, a chronic medical illness: implications for treatment, insurance, and outcomes evaluation. JAMA 284:1719-1720

3. Ouimette P, Moos R, Finney J (1998) Influence of outpatient treatment and 12-step group involvement in one-year substance abuse treatment outcomes. J Stud Alcohol 59:513-522

4. Vaillant G (1996) A long-term follow-up of male alcohol abuse. Arch Gen Psychiatry 53:243-249

5. Vuchinich R, Tucker J (1996) Alcoholic relapse, life events, and behavioral theories of choice: a prospective analysis. Exp Clinical Psychopharmacol 4:19-28

6. Bouton M (2000) A learning theory perspective on lapse, relapse, and the maintenance of behavior change. Health Psychology 19:57-63

7. Shalev U, Grimm J, Shaham Y (2002) Neurobiology of relapse to heroin and cocaine seeking: a review. Pharmacol Rev 54:1-42

8. Shaham Y, Shalev U, Lu L, Harrier W, Stewart J (2003) The reinstatement model of drug relapse: history, methodology, and major findings. Psychopharmacology 168:3-20

9. Stone A, Shiffman S (1994) Ecological momentary assessment (EMA) in behavioral medicine. Annals of Behavioral Medicine 16:199-202

10. Simpson D, Joe G, Fletcher B, Hubbard R, Anglin M (1999) A national evaluation of treatment outcomes for cocaine dependence. Arch Gen Psychiatry 56:516-518

11. Fogg B (2002) Interactive computing systems designed to change people's attitudes and behaviors. Stanford University Press, Palo Alto

12. Picard R, Liu K (2007) Relative subjective count and assessment of interruptive technologies applied to mobile monitoring of stress. Int J Human-Computer Studies 65:371-375
13. Picard R, Healy J (1997) Affective wearables. Personal Technologies 11:231-240

14. Picard R, Liu K (2004) A personal, mobile system for understanding stress and interruptions. M.S. Thesis, MIT Media Arts and Science

15. Murphy K (2002) Dynamic Bayesian Networks: Representation, inference and learning. PhD dissertation, UC Berkeley

16. Pearl J (1988) Probabilstic reasoning in intelligent systems. Morgan Kaufmann, San Mateo, CA

17. Fogarty L, Roter D, Larson S, Levy R (2002) Patient adherence to HIV medication regimens: a review of published and abstrcdt reports. Patient Education and Counseling 46:93-108

18. DunbarJacob J, Burke L, Puczynski S (1995) Clinical assessment and management of adherence to medical regimens. In: NPaS TW (ed) Managing chronic illness. American Psychological Association, Washington, DC, pp 313-350

19. Murphy D, Roberts K, Martin D, Marelich W (2000) Barriers to antiretroviral adherence amon $\mathrm{HIV}$-infected adults. Aids patient care and STDs 14:47-58

20. Malta M, Magnani M, Strathdee S, Bastos F (2008) Adherence to antiretroviral therapy among HIV-infected drug users: a metaanalysis. AIDS and Behavior. Available at http://www.spring erlink.com/content/h678m56641730350/.

21. (2010) Technology, HAART adherence and substance abuse. In; National Institute on Drug Abuse, Washington, DC, March 4-5

22. Epstein D, WillnerReid J, Vahabzadeh M, Mezghanni M, Preston K (2009) Real-time electronic diary reports of cue exposure and mood in the hours before cocaine and heroin craving and use. Arch Gen Psychiatry 66:88-94

23. Curry S, Mermelstein R, Sporer A (2009) Therapy for specific problems: youth tobacco cessation. Annual Review of Psychology 60:229-255

24. Safren S, Otto M, Worth J et al (2001) Two strategies to increase adherence to HIV antiretroviral medication: Life-Steps and medication monitoring. Behavior Research and Therapy 39: $1151-1162$

25. Fischer J, Bryan A, Fisher W, Misovich S (2002) InformationMotivation-Behavioral skills model-based HIV risk behavior change intervention for inner-city high school youth. Health Psychology 21:177-186

26. Rhodes F, Malotte K. Using stages of change to assess intervention readiness and outcome in modifying drug-related and sexual HIV risk behaviors in IDUs and crack users. In: Trotter R (ed) Multicultural AIDS prevention programs. Haworth Press, Binghampton, NY

27. Evans A, Edmundson-Drane E, Harris K (2000) Computerassisted instruction: an effective instructional method for HIV prevention education. Journal of Adolescent Health 26:244-251 